\title{
Gossypiboma: A Dramatic Result of Human Error, Case Report and Literature Review
}

\author{
Yusuf Arıkan, Osman Ozdemir, Kamil Gokhan Seker, Mithat Eksi, \\ Ekrem Guner, Nadir Kalfazade, Selcuk Sahin, Ali Ihsan Tasci \\ Department of Urology, Bakirkoy Dr. Sadi Konuk Training and Research Hospital, \\ Istanbul, Turkey \\ Received April 14, 2019; Accepted December 1, 2019.
}

Key words: Gossypiboma - Foreign body - Renal cell carcinoma - Bladder cancer

Abstract: Gossypiboma refers to a retained foreign object that was forgotten in the body cavity during an operation. It is a rare surgical complication that most commonly occurs after intraperitoneal abdominal emergency surgical procedures, but may also occur after virtually any type of operation. Gossypiboma can be confused with neoplastic lesions and abscess. Clinical examination and radiological findings may sometimes mislead the physician. We intend to present our cases, which is thought to be a kidney tumour and bladder cancer but resulted gossypiboma which is a condition that is caused by a forgotten sponge during the operation and it can mimic the cancer. During the operation, the team must work in coordination and be careful. Unnecessary operations in such situation can significantly increase the patient's morbidity.

Mailing Address: Yusuf Arikan, MD., Department of Urology, Bakirkoy

Dr. Sadi Konuk Training and Research Hospital, Tevfik Saglam Caddesi No. 11 Zuhuratbaba/Bakirkoy, Istanbul 34147, Turkey; Phone: +900 54220493 28; e-mail: dryusufarikan@gmail.com 


\section{Introduction}

Gossypiboma is a rare situation caused by the forgetting a surgical sponge in the body cavities during a surgical operation and consequently by the reaction of the body to foreign matter. The term "gossypiboma" is derived from the Latin gossypium (cotton wool, cotton) and the suffix -oma, meaning a tumour or growth, and describes a mass within a patient's body comprising a cotton matrix surrounded by a foreign body granuloma (Alayo et al., 2010).

Although gossypiboma is a lesion that can mimic benign or malignant tumours or abscess, its diagnosis can usually be done years after the surgery. On the other hand, it is rarely reported for medicolegal reasons (Manzella et al., 2009).

"Emergency operation" and "poor communication" were stated as the risk factors of forgetting a surgical sponge, which is the cause of the gossypiboma (Wan et al., 2009).

Patients may present with nonspecific symptoms that are the result of a foreign body related inflammatory process, or they may be asymptomatic and be diagnosed incidentally (Dakubo et al., 2009).

\section{Case report}

Case 1

During routine controls of a 74-year-old man, an approximately $5 \times 5 \mathrm{~cm}$ in diameter mass lesion was detected in the middle pole of the left kidney. The patient's comorbidities included hypertension (HT), polycystic kidney disease and chronic renal failure (CRF). He had a history of left open pyelolithotomy and cholecystectomy 20 years ago. MRI (magnetic resonance imaging) showed a $5.5 \times 4.5 \mathrm{~cm}$ in diameter smooth contoured contrast enhanced lesion with an exophytic extension in the mid-lower section of the left kidney (Figure 1). Left
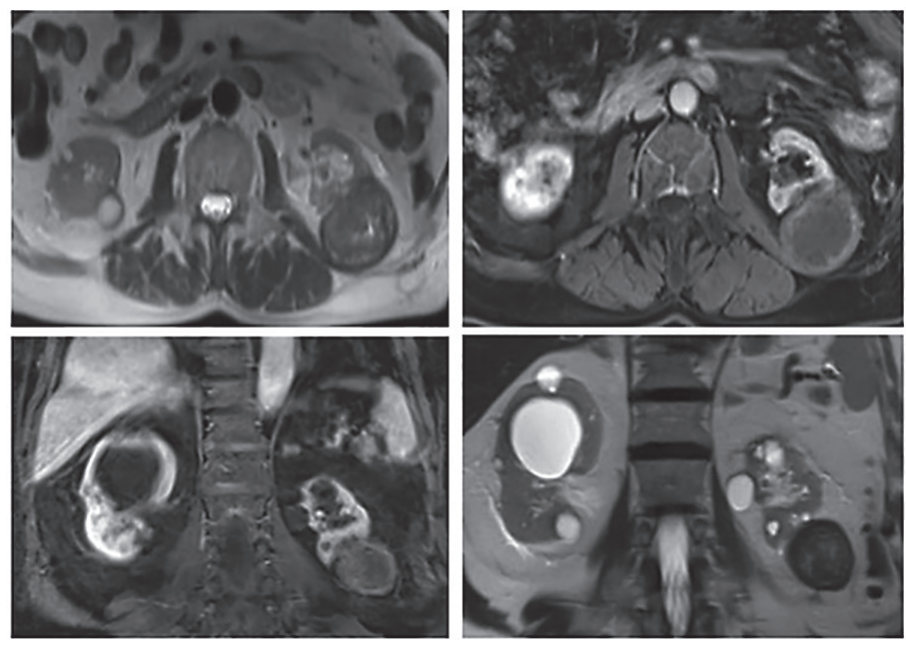

Figure 1 - A contrastenhanced magnetic resonance imaging scan of lesion in the posterior pole of the left kidney. 


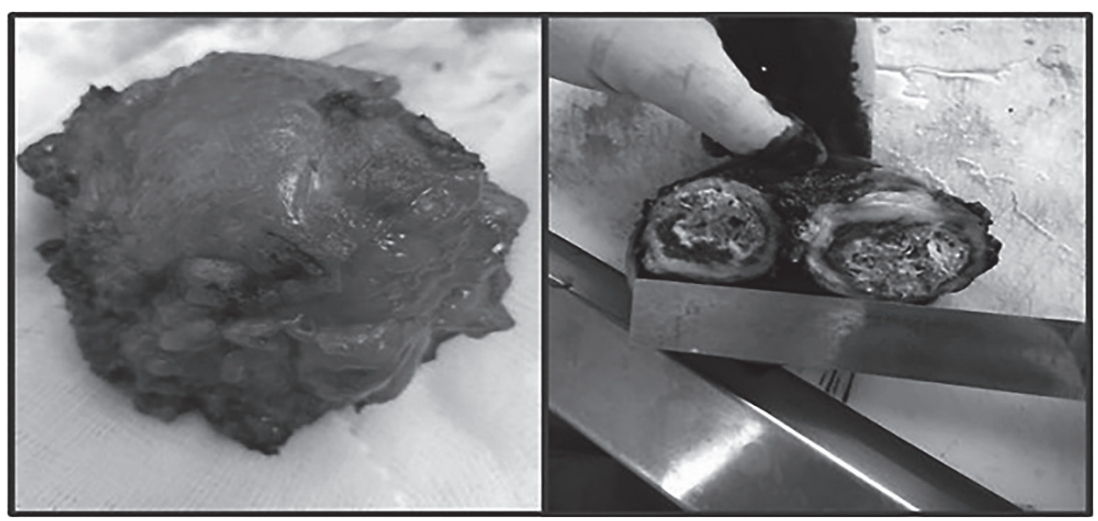

Figure 2 - The macroscopic images of pathological specimen.
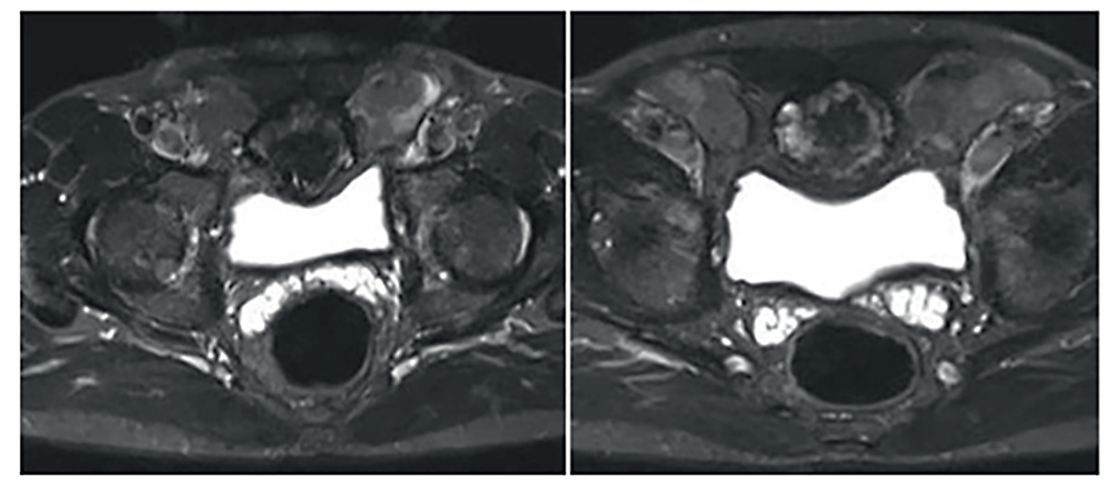

Figure 3 - A contrast-enhanced computed tomography scan of the lesion in the bladder anterior wall that induced to bladder.

partial nephrectomy was performed in the patient with suspicious appearance for malignancy. The macroscopic pathological specimen is shown in Figure 2.

Microscopic examination of partial nephrectomy material showed fibrinous material in the cytoplasm of histiocytes, some of which are multinucleated. The pathology report resulted as foreign body type chronic inflammatory granulation tissue. It was thought that was a result of forgotten surgical sponge in the operation area due to open left pyelolithotomy 20 years ago.

\section{Case 2}

A 52-year-old woman presented with dysuria and frequent urination. The patient had no chronic disease. She had a history of open cystolithotomy six years ago. Imaging showed a $45 \times 42 \mathrm{~mm}$ in diameter heterogeneous contrast enhanced mass lesion in mesentery tissue in the bladder dome (Figure 3). Cystoscopic examination showed protruding and encrusted threads that might be a part of impacted surgical 
gauze at the dome of the bladder. Pelvic exploration and foreign body removal were performed. Pathology results were reported as active chronic inflammation with foreign body type giant cells. It was thought that the patient was presented in this way due to the gauze that had been forgotten during an open cystolithotomy operation 6 years ago.

\section{Discussion}

Gossypiboma is not accurately reported for medico-legal reasons and its actual incidence is difficult to predict. However, there are publications indicating that the frequency is 1 in 4,000 (Kiernan et al., 2008). In another publication, it was reported that the ratio ranged from 1 in 1,000 and 1 in 300 cases in all surgical cases, and between 1 in 1,500 and 1 in 1,000 cases in intraabdominal cases (Lincourt et al., 2007).

Complaints of patients with gossypiboma depend on the inflammatory process of the body against foreign bodies (Dakubo et al., 2009). There are different responses in the early and late periods, depending on the forgotten material. In the early period, problems such as abscesses and infections occur, but in the late period, problems such as obstruction and chronic pain due to adhesions are encountered (Sakorafas et al., 2010). Gomes et al. (2017) described gossypiboma as a lifethreatening surgical site infection and stated that this might be a cause of sepsis.

The patients can be completely asymptomatic and may be diagnosed incidentally, but gossypiboma may also appear with complaints similar to intestinal obstruction, intestinal fistula, malabsorption syndrome or diffuse peritonitis (Iglesias and Salomao, 2007). It's been stated that gossypiboma is a lesion that can mimic benign or malignant tumours and abscess (Manzella et al., 2009). In one of the cases we presented, the patient was completely asymptomatic and underwent surgery with a preliminary diagnosis of renal tumour. In the other case, the patient presented with lower urinary tract symptoms and was diagnosed with cystoscopy.

Parashari et al. (2012) stated that gauze forgetting is an important and preventable cause of morbidity. In a review of 254 patients published by Wan et al. (2009), it was concluded that the most important predisposing factors of gauze forgetting are "emergency operation" and "poor communication". When we look at these studies, gossypiboma is a widely preventable condition in the literature and is the result of an absolute human error.

Gossypiboma is not a frequently diagnosed lesion and requires clinician's suspicion (Apter et al., 1990). In patients with long-standing nonspecific symptoms, the history of the patient should also be questioned and especially if there is a history of previous surgery, gossypiboma should be considered (Karasaki et al., 2013; Rehman et al., 2014).

Gossypiboma appears as a spongy structure with characteristic gas bubbles in radiological examinations. Calcification and contrast enhancement may also occur in the lesion wall (O'Connor et al., 2003). 
In the medicolegal aspect, gossypiboma is a medical negligence and it can be avoidable. In the surgeon's side it can cause humiliation, mental suffering or maybe charges for malpractice. In the patients side it may cause increased morbidity or mortality and this situation can cause medical and legal problems between surgeon and patients (Biswas et al., 2012).

The final treatment of gossypiboma is described as surgical operation and removal of foreign body. Today, the gauze count is shown as the gold standard to prevent gossypiboma and to avoid from gauze forgetting (Rehman et al., 2017).

\section{Conclusion}

Gossypiboma is an unpleasant condition that occurs due to a forgotten foreign body in the body during operation. It sometimes causes no symptoms at all, and in some cases mimics the tumour, may cause indeterminate pain in patients, and may be the cause of sepsis and cause of death.

When the diagnosis of gossypiboma is made, the absolute solution is the removal of the foreign body with surgery and the gauze must be counted in every operation to prevent gossypiboma. The best approach to prevent such problems requires the operation team to have appropriate communication with each other to avoid situations that cause inattention.

\section{References}

Alayo, E., Attar, B., Go, B. (2010) A case of recurrent abdominal pain due to a gossypiboma with spontaneous resolution. Clin. Gastroenterol. Hepatol. 8, e13-e14.

Apter, S., Hertz, M., Rubinstein, Z. J., Zissin, R. (1990) Gossypiboma in the early post-operative period: a diagnostic problem. Clin. Radiol. 42, 128-129.

Biswas, R. S., Ganguly, S., Saha, M. L., Saha, S., Mukherjee, S., Ayaz, A. (2012) Gossypiboma and surgeon Current medicolegal aspect - a review. Indian J. Surg. 74(4), 318-322.

Dakubo, J., Clegg-Lamptey, J., Hodasi, W., Obaka, H., Toboh, H., Asempa, W. (2009) An intra-abdominal gossypiboma. Ghana Med. J. 43(1), 43-45.

Gomes, C. A., Catena, F., Soares, C. Jr., Sartelli, M., di Saverio, S., Gomes, F. C., Gomes, C. C. (2017) Septic abdominal gossypiboma: A life threatening surgical site infection. JoPER 2, 70.

Iglesias, A. C., Salomao, R. M. (2007) Gossipiboma intra-abdominal - Análise de 15 casos. Rev. Col. Bras. Cir. 34, 106-113. (in Portuguese)

Karasaki, T., Nomura, Y., Nakagawa, T., Tanaka, N. (2013) Beware of gossypibomas. BMJ Case Rep. 2013.

Kiernan, F., Joyce, M., Byrnes, C. K., O’Grady, H., Keane, F. B., Neary, P. (2008) Gossypiboma: A case report and review of the literature. Ir. J. Med. Sci. 177, 389-391.

Lincourt, A. E., Harrell, A., Cristiano, J., Sechrist, C., Kercher, K., Heniford, B. T. (2007) Retained foreign bodies after surgery. J. Surg. Res. 138, 170-174.

Manzella, A., Filho, P. B., Albuquerque, E., Farias, F., Kaercher, J. (2009) Imaging of gossypibomas: pictorial review. AJR Am. J. Roentgenol. 193, S94-S101 (Suppl. 6).

O'Connor, A. R., Coakley, F. V., Meng, M. V., Eberhardt, S. (2003) Imaging of retained surgical sponges in the abdomen and pelvis. AJR Am. J. Roentgenol. 180, 481-489.

Parashari, U. C., Khanduri, S., Saxena, S., Bhadury, S. (2012) Gossypiboma: An important preventable cause of morbidity. Ann. Trop. Med. Public Health 5, 397-399.

Arıkan Y.; Ozdemir O.; Seker K. G.; Eksi M.; Guner E.; Kalfazade N.; Sahin S.; Tasci A. I. 
Rehman, A., Baloch, N. U., Awais, M. (2014) Gossypiboma diagnosed fifteen years after a cesarean section: a case report. Qatar Med. J. 2014, 65-69.

Rehman, A., Ul-Ain Baloch, N., Awais, M. (2017) Gossypiboma (retained surgical sponge): an evidence-based review. Open J. Surg. 1(1), 8-14.

Sakorafas, G. H., Sampanis, D., Lappas, C., Papantoni, E., Christodoulou, S., Mastoraki, A., Safioleas, M.

(2010) Retained surgical sponges: What the practicing clinician should know. Langenbecks Arch. Surg. 395 , 1001-1007.

Wan, W., Le, T., Riskin, L., Macario, A. (2009) Improving safety in the operating room: A systematic literature review of retained surgical sponges. Curr. Opin. Anaesthesiol. 22, 207-214. 